\title{
Analysis of the Signal Transfer and Folding in N-path Filters with a Series Inductance
}

\author{
Lammert Duipmans, Remko Struiksma, Eric A.M. Klumperink, Senior Member, IEEE, Bram Nauta, \\ Fellow, IEEE, Frank E. van Vliet, Senior Member, IEEE ${ }^{1}$
}

\begin{abstract}
N-path filters exploiting switched-series-R-C networks can realize high- $Q$ blocking-tolerant band-pass filters. Moreover, their center frequency is flexibly programmable by a digital clock. Unfortunately, the time variant nature of these circuits also results in unwanted signal folding. This paper proves analytically that folding can be reduced and band pass filtering can be improved by adding an inductance in series to the switched-R-C network. In contrast, a shunt capacitor degrades band-pass filter performance. The interaction between the reactive series impedance and the switched capacitors of an $\mathrm{N}$-path filter complicates analysis due to memory effects associated with reactive components. Assuming $\mathbf{N}$ identical signal paths with $1 / \mathrm{N}$ duty cycle, we show it is possible to solve the set of differential equations, by assuming that the signals in each path only differ in delay. Analytical equations are verified versus simulations, and the benefits in filter properties and reduction in signal folding are demonstrated.
\end{abstract}

Index Terms-Filter, N-path filter, frequency translated filtering, switched capacitor filters, commutated network filters, tunable filter, software defined radio, cognitive radio, reconfigurable filter, Linear Periodically Time Variant Circuit.

\section{INTRODUCTION}

$\mathrm{T}$ UNABLE filters with high linearity and strong blocker handling capability are highly wanted to realize reconfigurable radio receivers and enable cognitive radio [1]. Recent research has shown that $\mathrm{N}$-path filters $(\mathrm{N} \geq 3)$ are promising candidates to realize tunable band-pass filters [2-12] and also simultaneous frequency and spatial domain filtering $[13,14]$. Although the concept dates back to the 60 s of the previous century $[15,16]$, only with the advent of nanometer CMOS technologies can such filters now operate at low $\mathrm{GHz}$ RF frequencies. Figure 1 shows a 4-path filter with 4 capacitors and switches driven by multi-phase non-overlapping digital clocks that define the filter center frequency. Basically the input signal is mixed down, filtered, and mixed up again by the same set of switches, resulting in second order band-pass filter behavior, also without the inductor: essentially the low-pass R$\mathrm{C}$ filter shape is shifted to around $f_{S}$. The associated bandwidth BW defined by the RC time and duty cycle of the clock can be as low as a few $\mathrm{MHz}$, equal to a typical channel bandwidth. As $\mathrm{GHz}$ clock frequencies are possible, the $Q=f_{S} / B W$ can be high, e.g. a $\mathrm{Q}>100$ is feasible. Moreover, the linearity of passive mixers realized with MOS switches can be very good, and blockers in the order of $0 \mathrm{dBm}$ can be handled provided the switches have sufficient overdrive voltage. As switches and capacitors scale well with CMOS downscaling, the technique

Paper submission: June 11th 2014; This research was conducted as part of the Sensor Technology Applied in Reconfigurable systems for sustainable Security (STARS) project funded by the Dutch Government. All authors are
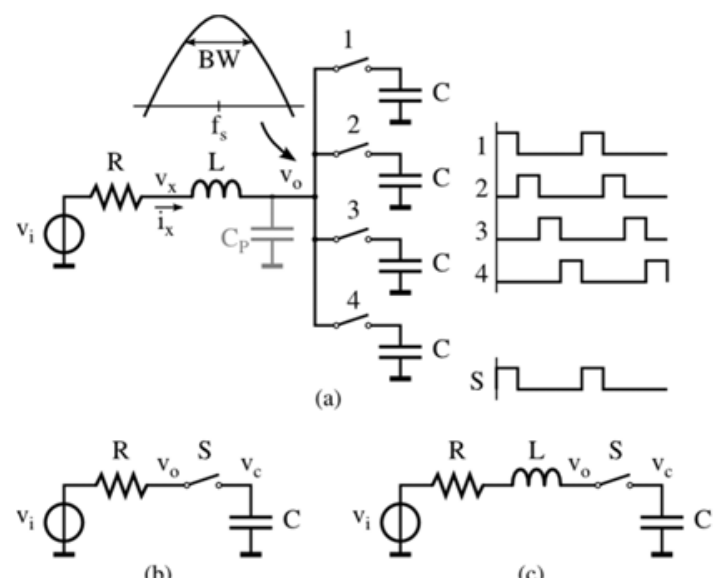

Figure 1: a) Single-ended 4-path switch-R-C filter with series inductor L and parasitic capacitance $C_{P}$. Switches are driven by non-overlapping clocks. Conditionally, the circuit can be split in independent single-ended "kernels", where: (b) is a switch-R-C kernel (index R); (c) is a switch-R-L-C kernel.

can benefit from process scaling (see for instance [14] which compares a $65 \mathrm{~nm}$ and $28 \mathrm{~nm}$ chip).

Unfortunately, the time variant nature of $\mathrm{N}$-path filters due to the on/off switching also results in unwanted signal folding (somewhat similar to aliasing in samplers, but with attenuation due to the embedded low-pass filtering as we will see later). Hence, pre-filtering is wanted to attenuate signals that would otherwise fold on top of the wanted signal. Pre-filtering can also reduce harmonic passbands at multiples of the switching frequency, although these are less problematic than folding (they do not overlap with the main filter passband). Although using more paths allows for cancelling more harmonics and folding products [17], there is a limit to the number of feasible phases for a multi-phase clock because of process speed and power consumption. Moreover, phase errors limit the achievable cancellation of harmonic and folding responses. Hence, assistance by a pre-filter may be needed to achieve sufficient suppression of a problematic folding product. The strongest folding to the passband of interest occurs from $(N-1) f_{S}$, the folding sideband nearest to the passband [17]. For a 4-path this is around $f_{R F} \approx 3 f_{S}$ and for 8 paths around $f_{R F} \approx 7 f_{S}$. As these frequencies are more than an octave away from the desired signal at $f_{R F} \approx f_{S}$, low-order low-pass prefiltering can be enough to attenuate folding products. However, filter design is less straightforward than one might expect. This is due to interaction between reactive pre-filter components

or have been with the University of Twente, P.O. Box 217, Enschede, The Netherlands (corresponding author e-mail: e.a.m.klumperink@utwente.nl). F.E. van Vliet is also at TNO, Den Haag, The Netherlands. 


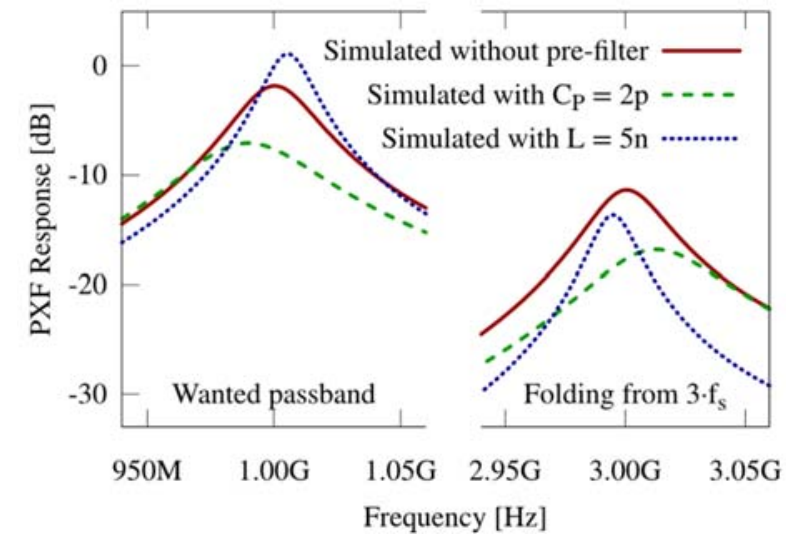

Figure 2 Simulated Periodic Transfer Function (PXF) for the wanted transfer and unwanted folding from $3 \mathrm{f}_{\mathrm{s}}$ for the 4-path filter of Figure 1a, showing improvement with a series inductor $\mathrm{L}\left(\mathrm{C}_{\mathrm{P}}=0\right)$ and degradation with a shunt capacitor $C_{P}(L=0)$, compared to the pre-filter less resistive case $\left(L=0, C_{P}=0\right)$.

with memory and the switched capacitors. The circuit in Figure 1a with resistive source $(\mathrm{L}=0)$ can be analyzed by exploiting the lack of memory in a resistor, and the lack of interaction between the capacitor charges due to the non-overlapping clocks. Hence the circuit can be split in independent kernels (Figure 1b [5, $18]$ ), and the response of the complete system can be found by adding the responses of the kernels. However, if an inductor or capacitor is present between the signal source and switches, the states of the switched capacitors are also affected by the mutual interaction via this memory element. The purpose of this paper is to derive analytical equations describing this interaction and its impact on signal transfer and folding. Although it has been observed that adding a series inductor can improve the filter transfer and noise $[19,20]$, only approximate equations for the desired transfer function, the input impedance and noise are available [4, 21, 22], assuming the RF-frequency is $f_{R F}=f_{S}+$ $\Delta f$, with $\Delta f \ll f_{L O}$. Broadband signal folding cannot be analyzed with these equations. This paper aims at extending the exact analysis in $[5,12,18]$ to include memory effects for the case that a series inductance is added. We focus on this case as, in contrast to adding a shunt capacitor $C_{P}$ before the switches, a series inductance increases passband gain and selectivity, while also reducing signal folding (see Figure 2).

This paper is structured as follows: in section II the analysis of the switched-series-R-C kernel in Figure $1 \mathrm{~b}$ is reviewed [5, 18]. This both serves as basis for the analysis of the case with series inductance (Figure 1c) in section III, and as benchmark to quantify filter improvements. Section IV compares analysis results with simulations and section $\mathrm{V}$ presents conclusions.

\section{N-PATH FILTER WITH RESISTIVE SOURCE IMPEDANCE}

In [18] a detailed analysis of a switched series R-C network is presented that is also applicable to $\mathrm{N}$-path filter analysis as shown in [5]. In this section, we quickly review the analysis for a resistive source impedance, as basis for the analysis with an inductive source impedance, which is done in the next section.

In a single-ended N-path filter the switches are driven by polyphase clocks, i.e. clocks with the same duty-cycle starting at regularly spaced intervals within the period time. The clocks do not overlap when high and the capacitor voltages are thus independent, which simplifies analysis. In Figure 3, a timing diagram of the intervals in a general N-path filter is shown, where $\sigma_{0}$ is defined to be 0 . To simplify mathematical expressions, ideal switches will be assumed that switch infinitely fast between $R_{\text {off }}$ of infinity and $R_{o n}$ of zero. Moreover, we assume that no time interval exists in which all switches are open, i.e. the clock duty-cycle is $1 / \mathrm{N}$, with $\mathrm{N}$ the number of paths. Thus only the switch in the k-th path will be closed during interval $\mathrm{k}$, during an on-time $\mathrm{T}_{\mathrm{s}} / \mathrm{N}$.

The analysis will now be performed by decomposing the $\mathrm{N}$ path circuit in so-called "kernels" [18] as shown in Figure 1b. First, only one kernel will be analyzed. Other kernels have the same transfer function, except for a phase shift in the clock. We can find the combined effect of all kernels in a polyphase system, using the analysis for one kernel and adding that phase shift term later, in subsection A. We choose the kernel for which the switch is closed during $\mathrm{k}=1$ for analysis, so $\tau_{1}$ is the on-time of the switch and $\tau_{2}$ the off-time, as shown in Figure $3 b$.

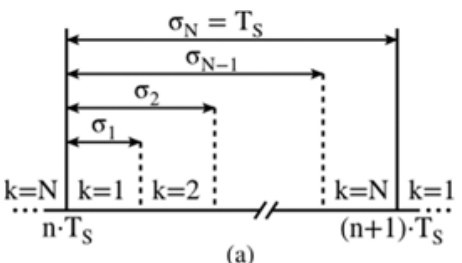

(a)

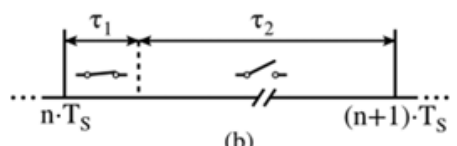

(b)

Figure 3 a) Timing diagram for (a) the N-path filter, (b) the kernel to be analyzed, which contributes to $\mathrm{v}_{\mathrm{o}}(\mathrm{t})$ during $\mathrm{k}=1$.

For zero switch resistance, the output voltage $v_{o}(t)$ is equal to one of the capacitor voltages. The kernel under analysis defines the output only during $\mathrm{k}=1$. Other kernels will provide the output during other intervals, each for exactly $1 / \mathrm{Nth}$ of $\mathrm{T}_{\mathrm{s}}$. This periodicity is crucial for the filter behavior and signal folding to be analyzed. In order to limit mathematical derivations, we will write equations in the same form as in $[18,23]$ and re-use the Fourier transform derived there [16]. To this end we will write differential equations as a function of $\mathrm{t}$ and $\mathrm{k}$, where $\mathrm{k}$ relates to the time variant behavior. Considering the kernel in Figure $1 \mathrm{~b}$ during an interval identified by $\mathrm{k}$, the following equation holds:

$$
\begin{gathered}
\frac{d v_{C}(t)}{d t}=A_{k, R} v_{c}(t)+C_{k, R} v_{i}(t), \\
n T_{s}+\sigma_{k-1} \leq t<n T_{s}+\sigma_{k}
\end{gathered}
$$

We have a series-R-C network (index R) only during k=1, so:

$$
A_{1, R}=-\frac{1}{R C}, \quad C_{1, R}=-A_{1, R}=\frac{1}{R C}
$$

while the coefficients are 0 for other $\mathrm{k}$ values (the capacitor voltage doesn't change during the hold-phase). Following [16] we introduce a time variant windowed version $v_{c, k}(t)$ of the capacitor voltage, defined as:

$$
v_{c, k}(t)=w_{k}(t) \cdot v_{c}(t)
$$

$w_{k}(t)=\left\{\begin{array}{l}1, \\ 0\end{array}\right.$

$$
\begin{gathered}
n T_{s}+\sigma_{k-1} \leq t<n T_{s}+\sigma_{k} \\
\text { elsewhere }
\end{gathered}
$$


where $w_{k}(t)$ is a time windowing function that is useful from a mathematical point of view [16], and conveniently chosen so that the contributions of $v_{c, k}(t)$ to the output voltage $v_{o}(t)$ occur during this time window. The window can be modelled by step functions and as derived in Appendix A, the differential equation for the kernel, valid for all $t$, can now be written as:

$$
\begin{aligned}
& \frac{d v_{c, k}(t)}{d t} \\
& \quad=A_{k, R} v_{c, k}(t)+C_{k, R} v_{i, k}(t) \\
& \quad+\sum_{n=-\infty}^{\infty} v_{c}(t)\left(\delta\left(t-n T_{s}-\sigma_{k-1}\right)-\delta\left(t-n T_{s}-\sigma_{k}\right)\right)
\end{aligned}
$$

where the $\delta$-functions add and subtract samples at interval ends, as discussed in ([23], eq.(7)). By applying the Fourier transform to this equation, $V_{c, k}(f)$ is found [[18], appendix A]:

$$
V_{c, k}(f)=\sum_{n=-\infty}^{\infty} H_{n, k, R}(f) V_{i}\left(f-n f_{s}\right)
$$

where $H_{n, k, R}(f)$ is the transfer function associated with frequency shift $n f_{s}$, while index R refers to a purely resistive source $(\mathrm{L}=0)$. Note that a frequency component $V_{i}(f)$ at the input not only contributes to $V_{c}(f)$ as in a Linear Time Invariant filter, but also renders frequency shifted terms $\left(V_{i}\left(f-n f_{s}\right)\right)$. This renders signal folding to the pass band if an input signal exists at frequency spacing $n f_{s}$ from the pass band, which should be minimized. Evaluating the Fourier transform gives [18]:

$$
\begin{aligned}
& H_{n, k, R}(f) \\
& \quad=\frac{1}{j 2 \pi f-A_{k, R}}\left(C_{k, R} \frac{1-e^{-j 2 \pi n / N}}{j 2 \pi n} e^{-j 2 \pi n f_{s} \sigma_{k-1}}\right. \\
& \quad+f_{s} G_{k-1, R}\left(f-n f_{s}\right) e^{-j 2 \pi n f_{s} \sigma_{k-1}} \\
& \left.\quad-f_{s} G_{k, R}\left(f-n f_{s}\right) e^{-j 2 \pi n f_{s} \sigma_{k}}\right)
\end{aligned}
$$

Because the kernels are identical, it is sufficient to determine the transfer of only one of them. As we analyzed a kernel active during $\mathrm{k}=1$, i.e. $H_{n, 1, R}(f)$. The functions $\mathrm{G}_{0, \mathrm{R}}$ and $\mathrm{G}_{1, \mathrm{R}}$ that are then required for eqn. (7) are [[18], app. B]:

$$
\begin{gathered}
G_{0, R}(f)=\frac{1}{1+j 2 \pi f R C}\left(\frac{e^{j 2 \pi f \tau_{1}}-e^{\frac{-\tau_{1}}{R C}}}{e^{j 2 \pi f T_{S}}-e^{\frac{-\tau_{1}}{R C}}}\right) \\
G_{1, R}(f)=G_{0, R}(f) e^{j 2 \pi f \tau_{2}}
\end{gathered}
$$

The function $G_{0, \mathrm{R}}(f)$ is the transfer to the output voltage when sampled at $n T_{S}$ (when the switch closes) and $G_{1, \mathrm{R}}(f)$ when sampled at $n T_{S}+\sigma_{1}$ (when the switch opens). Because during $\tau_{2}$ (when the switch is open) the voltage on the capacitor does not change, these transfers differ only by a delay.

\section{A. Poly phase multi-kernel combination}

The final step is to combine the kernel transfer functions into one transfer function for the polyphase system. Assuming no switch resistance, kernel 1 , active during $\mathrm{k}=1$, contributes to $V_{o}(f)$ via transfer function $H_{n, 1, R}(f)$. The only difference between the kernels or paths in an N-path filter as depicted in Figure 1 is the phase shift of the clocks driving the switches. With $l$ defined as the path number and $\mathrm{N}$ the number of paths, the phase shift of the clock of kernel $l$ compared to kernel 1 is
$2 \pi \cdot(l-1) / N$. The transfer function of the combined output then is equal to the following sum [18]:

$$
\begin{aligned}
& H_{n, \text { polyphase }, R}(f) \\
& =\sum_{l=1}^{N} H_{n, 1, R}(f) e^{-\frac{j 2 \pi n(l-1)}{N}} \\
& =\left\{\begin{array}{cc}
0, & n / N \neq \text { integer } \\
N \cdot H_{n, 1, R}(f), & n / N=\text { integer }
\end{array}\right.
\end{aligned}
$$

with $\mathrm{H}_{\mathrm{n}, 1, \mathrm{R}}(\mathrm{f})$ the transfer of a single kernel calculated for the interval where the switch is on (note the periodicity with $\mathrm{N}$ ). The output spectrum is given by:

$$
V_{o}(f)=\sum_{n=-\infty}^{\infty} H_{n, \text { polyphase }, R}(f) V_{i}\left(f-n f_{s}\right)
$$

It can be seen from equation (10) that $H_{n, \text { polyphase } R}(f)$ is non-zero for $\mathrm{n}=\mathrm{kN}$, with $\mathrm{k}$ an integer. Hence, some of the harmonic folding terms remain un-cancelled, namely from input frequencies around $f_{s}-k N f_{s}$ to the desired filter band around $f_{s}$. Equations (7) and (10) can be combined and simplified. The transfer function with $\mathrm{n}=0$ (no frequency shift) can be determined by taking the limit of the first term between brackets in (7) for $n$ approaching zero. This leads to:

$$
\begin{aligned}
& \begin{array}{l}
H_{0, \text { polyphase }, R}(f) \\
=\frac{N}{j 2 \pi f+\frac{1}{R C}}\left(\frac{1}{N R C}+f_{S} \cdot\left(G_{0, R}(f)-G_{1, R}(f)\right)\right)
\end{array} \\
& =H_{R}(f)\left(1+\frac{1}{\Gamma_{R}}\left(G_{0, R}(f)-G_{1, R}(f)\right)\right) \\
& \text { Where: } \quad H_{R}(f)=\frac{1}{1+j 2 \pi f R C} \\
& \qquad \Gamma_{R}=\frac{T_{S}}{N R C}
\end{aligned}
$$

$\Gamma_{R}$ is the ratio between the switch-on time and the RC-time constant as introduced in [18], with the only difference that the duty-cycle is fixed here to $1 / \mathrm{N}$ to minimize signal loss [5]. For N-path filters, the $Q=\frac{2 \pi f_{S}}{B W}=\frac{2 \pi}{\Gamma_{R}}$, so for high Q we want $\Gamma_{R} \ll$ $2 \pi$. Hence, we operate in the "slow" region [18], in which the $\mathrm{RC}$ time is much larger than the switch-on time (narrowband filtering, slow settling). Note that this is quite different from discrete-time switched-capacitor filters, where fast settling of capacitor voltages is required within one clock period.

The harmonic folding effects are characterized by equations (8) and (9) with $\mathrm{n}$ non-equal to zero. Taking into consideration that the on-time is always $\mathrm{T}_{\mathrm{s}} / \mathrm{N}$ and $\sigma_{0}$ is 0 we find:

$$
\begin{gathered}
H_{n, \text { polyphase }, R}(f) \\
=\frac{H_{R}(f)}{\Gamma_{R}} \cdot\left(G_{0, R}\left(f-n f_{s}\right)-G_{1, R}\left(f-n f_{S}\right)\right), \\
n / N=\text { integer and } n \neq 0
\end{gathered}
$$

\section{N-PATH FILTER With Inductive SOURCE IMPEDANCE}

In the current section, an N-path filter with a series inductance as shown in Figure 1a is analyzed. The presence of such a 'memory-element' results in interaction between the different paths. At the moment a switch closes, the inductor already carries a current which is dependent on the history of 
the voltage across the inductor, i.e. the previous path voltages.

The analysis starts again with decomposing the circuit into kernels (Figure 1c). However, now the differential equation valid during on-time $\tau_{1}$ of the switch is of second order:

$$
\begin{gathered}
\frac{d^{2} v_{c}(t)}{d t^{2}}=A_{k} v_{c}(t)+B_{k} \frac{d v_{c}(t)}{d t}+C_{k} v_{i}(t), \\
n T_{s}+\sigma_{k-1} \leq t<n T_{s}+\sigma_{k}
\end{gathered}
$$

With: $A_{1}=-C_{1}=\frac{-1}{L C}, \quad B_{1}=-\frac{R}{L}$

while the coefficients are zero for other values of $\mathrm{k}$. The voltage transfer function can be found by applying the Fourier transform on the differential equation above. In order to make this possible, the equation has to be made valid for all $\mathrm{t}$. This process is described in Appendix A and results in:

$$
\begin{aligned}
& \frac{d^{2} v_{c, k}(t)}{d t^{2}} \\
& =A_{k} v_{c, k}(t)+B_{k} \frac{d v_{c, k}(t)}{d t}+C_{k} v_{i, k}(t) \\
& \quad+\sum_{n=-\infty}^{\infty}\left\{-B_{k} v_{c}(t)\left(\delta\left(t-n T_{s}-\sigma_{k-1}\right)\right.\right. \\
& \left.-\delta\left(t-n T_{s}-\sigma_{k}\right)\right) \\
& +\frac{d\left(v_{c}(t)\left(\delta\left(t-n T_{s}-\sigma_{k-1}\right)-\delta\left(t-n T_{s}-\sigma_{k}\right)\right)\right)}{d t} \\
& \left.+\frac{d v_{c}(t)}{d t}\left(\delta\left(t-n T_{s}-\sigma_{k-1}\right)-\delta\left(t-n T_{s}-\sigma_{k}\right)\right)\right\}
\end{aligned}
$$

For evaluating the above equation, the sampled output voltage terms at the switching moments are needed and also the derivative of the output voltage at those moments. In the next section, the process of how to express these terms as a function of the input voltage will be described. When these terms are known, the Fourier transform can be performed on equation (18) leading to the wanted transfer function.

For sinusoidal input voltage $\mathrm{e}^{\mathrm{j} 2 \pi \mathrm{ft}}$ the general solution to equation (16) is of the form [24]:

$$
v_{c}(t)=c_{1} e^{s_{1}\left(t-t_{0}\right)}+c_{2} e^{s_{2}\left(t-t_{0}\right)}+c_{3} e^{j 2 \pi f\left(t-t_{0}\right)}
$$

in which the first two terms are the solution of the homogeneous equation, with $s_{1}$ and $s_{2}$ the roots of the characteristic polynomial [[24], Lesson 20]:

$$
s_{1,2}=\frac{B_{k}}{2} \pm \sqrt{\left(\frac{B_{k}}{2}\right)^{2}+A_{k}}=-\frac{R}{2 L} \pm \sqrt{\left(\frac{R}{2 L}\right)^{2}-\frac{1}{L C}}
$$

The constants will be solved from the initial conditions. The last term in equation (19) represents the particular solution [[24], lesson 21]. Substitution of this solution in (16) and evaluation for $\mathrm{t}=\mathrm{t}_{0}$ leads to:

$$
\begin{aligned}
C_{3} & =e^{j 2 \pi f t_{0}} \frac{C_{k}}{-A_{k}-j 2 \pi f B_{k}-(2 \pi f)^{2}} \\
& =e^{j 2 \pi f t_{0}} \cdot H(f)
\end{aligned}
$$

For $\mathrm{k}=1$ :

$$
H(f)=\frac{1}{1+j 2 \pi f R C-(2 \pi f)^{2} L C}
$$

The function $\mathrm{H}(\mathrm{f})$ is equal to the frequency domain transfer function $V_{c}(f) / V_{i}(f)$ of a single kernel with the switch closed.

The expression of the output voltage during the on-time of the switch is dependent on the initial conditions, i.e. the output voltage at the time instant the particular switch closes and its derivative (or strictly speaking the related current through the inductor). Suppose, the time instant of the closing of the switch is $t_{0}$. The remaining constants can now be determined by solving the following two equations for $\mathrm{c}_{1}$ and $\mathrm{c}_{2}$ :

$$
\begin{gathered}
v_{c}\left(t_{0}\right)=c_{1}+c_{2}+c_{3} \\
v_{c}^{\prime}\left(t_{0}\right)=c_{1} s_{1}+c_{2} s_{2}+c_{3} j 2 \pi f
\end{gathered}
$$

Resulting in:

$$
\begin{aligned}
& c_{1}=v_{c}\left(t_{0}\right) \frac{s_{2}}{s_{2}-s_{1}}-v_{c}^{\prime}\left(t_{0}\right) \frac{1}{s_{2}-s_{1}}+c_{3} \frac{j 2 \pi f-s_{2}}{s_{2}-s_{1}} \\
& c_{2}=v_{c}\left(t_{0}\right) \frac{-s_{1}}{s_{2}-s_{1}}+v_{c}^{\prime}\left(t_{0}\right) \frac{1}{s_{2}-s_{1}}+c_{3} \frac{s_{1}-j 2 \pi f}{s_{2}-s_{1}}
\end{aligned}
$$

Substitution of the constants in equation (19) gives:

$$
\begin{aligned}
& v_{c}(t) \\
& \quad=v_{c}\left(t_{0}\right) \frac{s_{2} e^{s_{1}\left(t-t_{0}\right)}-s_{1} e^{s_{2}\left(t-t_{0}\right)}}{s_{2}-s_{1}} \\
& +v_{c}^{\prime}\left(t_{0}\right) \frac{e^{s_{2}\left(t-t_{0}\right)}-e^{s_{1}\left(t-t_{0}\right)}}{s_{2}-s_{1}} \\
& +e^{j 2 \pi f t_{0}} H(f)\left(e^{j 2 \pi f\left(t-t_{0}\right)}-e^{s_{1}\left(t-t_{0}\right)}\right. \\
& \left.+\frac{e^{s_{2}\left(t-t_{0}\right)}-e^{s_{1}\left(t-t_{0}\right)}}{s_{2}-s_{1}}\left(s_{1}-j 2 \pi f\right)\right)
\end{aligned}
$$

And for the derivative of the output voltage: $v_{c}^{\prime}(t)$

$$
\begin{aligned}
& =v_{c}\left(t_{0}\right) \frac{s_{1} s_{2} e^{s_{1}\left(t-t_{0}\right)}-s_{1} s_{2} e^{s_{2}\left(t-t_{0}\right)}}{s_{2}-s_{1}} \\
& +v_{c}^{\prime}\left(t_{0}\right) \frac{s_{2} e^{s_{2}\left(t-t_{0}\right)}-s_{1} e^{s_{1}\left(t-t_{0}\right)}}{s_{2}-s_{1}} \\
& +e^{j 2 \pi f t_{0}} H(f)\left(j 2 \pi f e^{j 2 \pi f\left(t-t_{0}\right)}-s_{1} e^{s_{1}\left(t-t_{0}\right)}\right. \\
& \left.+\frac{s_{2} e^{s_{2}\left(t-t_{0}\right)}-s_{1} e^{s_{1}\left(t-t_{0}\right)}}{s_{2}-s_{1}}\left(s_{1}-j 2 \pi f\right)\right)
\end{aligned}
$$

Using these equations, the output voltage and the derivative of the output voltage at the end of the on-time of the switch can be calculated, given the initial conditions. Setting the time instant of closing of the switch at $t=n T_{s}$ and assuming that the on-time of the switch is equal to $\tau_{1}$ (see Figure 3), the following equations can be composed:

$$
\begin{aligned}
& \quad \begin{array}{l}
v_{c}\left(n T_{s}+\sigma_{1}\right) \\
=\alpha \cdot v_{c}\left(n T_{s}\right)+\beta \cdot v_{c}^{\prime}\left(n T_{s}\right)+\gamma \cdot e^{j 2 \pi f n T_{s}}
\end{array} \\
& \begin{array}{l}
v_{c}^{\prime}\left(n T_{s}+\sigma_{1}\right) \\
=\alpha^{\prime} \cdot v_{c}\left(n T_{s}\right)+\beta^{\prime} \cdot v_{c}^{\prime}\left(n T_{s}\right)+\gamma^{\prime} \cdot e^{j 2 \pi f n T_{s}} \\
\alpha=\frac{s_{2} e^{s_{1} \tau_{1}}-s_{1} e^{s_{2} \tau_{1}}}{s_{2}-s_{1}}
\end{array}
\end{aligned}
$$




$$
\begin{gathered}
\beta=\frac{e^{s_{2} \tau_{1}}-e^{s_{1} \tau_{1}}}{s_{2}-s_{1}} \\
\alpha^{\prime}=\frac{s_{1} s_{2} e^{s_{1} \tau_{1}}-s_{1} s_{2} e^{s_{2} \tau_{1}}}{s_{2}-s_{1}} \\
\beta^{\prime}=\frac{s_{2} e^{s_{2} \tau_{1}}-s_{1} e^{s_{1} \tau_{1}}}{s_{2}-s_{1}} \\
\gamma(f)=H(f)\left(e^{j 2 \pi f \tau_{1}}-e^{s_{1} \tau_{1}}+\beta \cdot\left(s_{1}-j 2 \pi f\right)\right) \\
\gamma^{\prime}(f) \\
=H(f)\left(j 2 \pi f e^{j 2 \pi f \tau_{1}}-s_{1} e^{s_{1} \tau_{1}}\right. \\
\left.+\beta^{\prime}\left(s_{1}-j 2 \pi f\right)\right)
\end{gathered}
$$

From now on, the process comes down to setting up the difference equations for the sampled $v_{c}$ and $v_{c}^{\prime}$. Since the capacitor voltage remains constant during the interval the switch is off, we also write:

$$
\begin{aligned}
& v_{c}\left((n+1) T_{s}\right) \\
& \quad=\alpha \cdot v_{c}\left(n T_{s}\right)+\beta \cdot v_{c}^{\prime}\left(n T_{s}\right)+\chi(f) e^{j 2 \pi f n T_{s}}
\end{aligned}
$$

For composing the difference equation for $v_{c}^{\prime}$, it is assumed that the current through the inductor at the moment the switch opens is equal to the current at the moment the switch of the next path closes. As a consequence, this also applies for the derivative of the output voltage at these time instants. With a duty-cycle of exactly $1 / \mathrm{N}$, this assumption will indeed be valid. Using this result:

$$
\begin{aligned}
& v_{c, l}^{\prime}\left((n+1) T_{s}\right) \\
& \quad=\alpha^{\prime} \cdot v_{c, l-1}\left((n+1) T_{s}-\frac{T_{s}}{N}\right) \\
& +\beta^{\prime} \cdot v_{c, l-1}^{\prime}\left((n+1) T_{s}-\frac{T_{s}}{N}\right) \\
& +\gamma^{\prime}(f) \cdot e^{j 2 \pi f\left((n+1) T_{s}-\frac{T_{s}}{N}\right)}
\end{aligned}
$$

In which $v_{c, l}$ is the capacitor voltage of path number $l$. At first sight, this difference equation seems difficult to solve, because it is dependent on the output state of the previous path, which is again dependent on the output state of the path before that, and so on. However, all paths are identical, albeit that the time interval in which they are connected to the output differs. As these intervals are spaced apart by $\mathrm{T}_{\mathrm{s}} / \mathrm{N}$, different paths will see a phase shifted version of the input signal. Assuming an input voltage $v_{i n}=e^{j 2 \pi f t}$, a relation between different path voltages of the following form should be expected:

$$
v_{c, l-1}\left((n+1) T_{s}-\frac{T_{s}}{N}\right)=v_{c, l}\left((n+1) T_{s}\right) e^{-\frac{j 2 \pi f T_{S}}{N}}
$$

A similar relation also holds for $v_{c}^{\prime}$ :

$$
v_{c, l-1}^{\prime}\left((n+1) T_{s}-\frac{T_{s}}{N}\right)=v_{c, l}^{\prime}\left((n+1) T_{s}\right) e^{-\frac{j 2 \pi f T_{s}}{N}}
$$

With help of the last two equations we can write (38) as:

$$
\begin{aligned}
& v_{c}^{\prime}\left((n+1) T_{s}\right) \\
& =\alpha^{\prime} \cdot e^{-\frac{j 2 \pi f T_{s}}{N}} v_{c}\left((n+1) T_{s}\right)+\beta^{\prime} \\
& \cdot e^{-\frac{j 2 \pi f T_{s}}{N}} v_{c}^{\prime}\left((n+1) T_{s}\right)+\gamma^{\prime}(f) \cdot e^{j 2 \pi f\left((n+1) T_{s}-\frac{T_{s}}{N}\right)}
\end{aligned}
$$

where the subscript $l$ is left out, because the function is no longer dependent on the path number. The next step is to solve for $v_{c}^{\prime}\left((n+1) T_{s}\right)$ and substituting $(n-1)$ for $n$ leading to:

$$
\begin{aligned}
& v_{c}^{\prime}\left(n T_{s}\right) \\
& =\frac{\alpha^{\prime}}{e^{\frac{j 2 \pi f T_{S}}{N}}-\beta^{\prime}} v_{c}\left(n T_{s}\right)+\frac{\gamma^{\prime}(f)}{e^{\frac{j 2 \pi f T_{S}}{N}}-\beta^{\prime}} e^{j 2 \pi f n T_{S}}
\end{aligned}
$$

Substituting this into equation (37) we get:

$$
\begin{aligned}
& v_{c}\left((n+1) T_{s}\right) \\
& \quad=\left(\alpha+\frac{\alpha^{\prime} \beta}{e^{\frac{j 2 \pi f T_{s}}{N}}-\beta^{\prime}}\right) v_{c}\left(n T_{s}\right) \\
& \quad+\left(\gamma(f)+\frac{\gamma^{\prime}(f) \cdot \beta}{e^{\frac{j 2 \pi f T_{S}}{N}}-\beta^{\prime}}\right) e^{j 2 \pi f n T_{s}}
\end{aligned}
$$

This equation is of the same form as [[18], eq. 87] and leads to the following steady state solution:

With:

$$
v_{c}\left(n T_{s}\right)=G_{0}(f) \cdot e^{j 2 \pi f n T_{s}}
$$

$$
G_{0}(f)=\frac{\gamma(f)+\frac{\gamma^{\prime}(f) \cdot \beta}{e^{\frac{j 2 \pi f T_{s}}{N}}-\beta^{\prime}}}{e^{j 2 \pi f T_{s}}-\left(\alpha+\frac{\alpha^{\prime} \beta}{e^{\frac{j 2 \pi f T_{s}}{N}}-\beta^{\prime}}\right)}
$$

Using (44), $v_{c}^{\prime}\left(n T_{s}\right)$ can be determined using equation (42):

With:

$$
v_{c}^{\prime}\left(n T_{s}\right)=I_{0}(f) \cdot e^{j 2 \pi f n T_{s}}
$$

$$
I_{0}(f)=\frac{\alpha^{\prime}}{e^{\frac{j 2 \pi f T_{S}}{N}}-\beta^{\prime}} G_{0}(f)+\frac{\gamma^{\prime}(f)}{e^{\frac{j 2 \pi f T_{s}}{N}}-\beta^{\prime}}
$$

Next, the goal is to find $\mathrm{G}_{1}$ and $\mathrm{I}_{1}$ (or equivalently: $v_{c}\left(n T_{s}+\sigma_{1}\right)$ and $\left.v_{c}^{\prime}\left(n T_{s}+\sigma_{1}\right)\right)$ :

$$
\begin{aligned}
& v_{c}\left(n T_{s}+\sigma_{1}\right) \\
& \quad=\alpha \cdot v_{c}\left(n T_{s}\right)+\beta \cdot v_{c}^{\prime}\left(n T_{s}\right)+\gamma(f) \cdot e^{j 2 \pi f n T_{s}} \\
& \quad=\left(\alpha \cdot G_{0}(f)+\beta \cdot I_{0}(f)+\gamma(f)\right) e^{j 2 \pi f n T_{s}}
\end{aligned}
$$

Hence:

$$
G_{1}(f)=\left(\alpha \cdot G_{0}(f)+\beta \cdot I_{0}(f)+\gamma(f)\right) \cdot e^{-\frac{j 2 \pi f T_{S}}{N}}
$$

When using equations (45) and (47) to evaluate (49), after some straightforward mathematics we get:

$$
G_{1}(f)=G_{0}(f) e^{j 2 \pi f\left(T_{S}-\frac{T_{S}}{N}\right)}
$$

Note that this relation was also found for the case without a reactive component. The procedure for finding $\mathrm{I}_{1}$ is similar:

$$
\begin{aligned}
& v_{c}^{\prime}\left(n T_{s}+\sigma_{1}\right) \\
& =\alpha^{\prime} \cdot v_{c}\left(n T_{s}\right)+\beta^{\prime} \cdot v_{c}^{\prime}\left(n T_{s}\right)+\gamma^{\prime}(f) \cdot e^{j 2 \pi f n T_{s}} \\
& =\left(\alpha^{\prime} \cdot G_{0}(f)+\beta^{\prime} \cdot I_{0}(f)+\gamma^{\prime}(f)\right) e^{j 2 \pi f n T_{s}} \\
& I_{1}(f)=\left(\alpha^{\prime} \cdot G_{0}(f)+\beta^{\prime} \cdot I_{0}(f)+\gamma^{\prime}(f)\right) \cdot e^{-\frac{j 2 \pi f T_{s}}{N}}
\end{aligned}
$$

After expressing $\mathrm{G}_{0}$ in terms of $\mathrm{I}_{0}$ by means of equation (47) and substitution in (52), the following simple result arises:

$$
I_{1}(f)=I_{0}(f)
$$




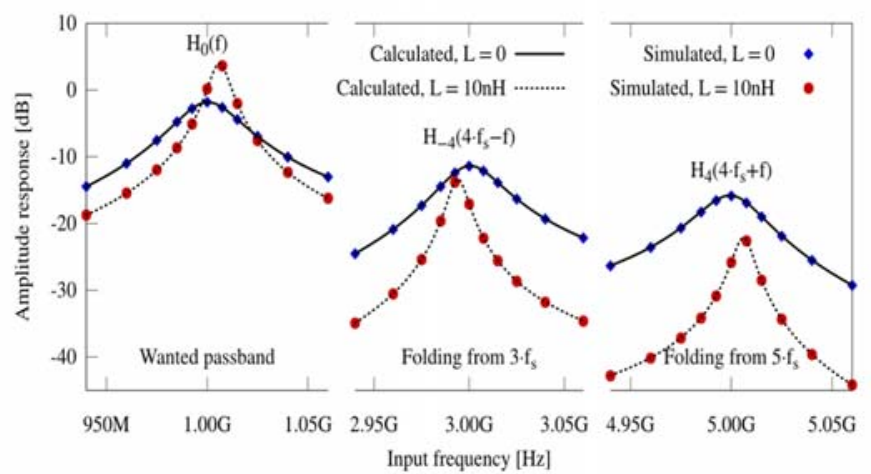

Figure 5 Calculated and simulated $H_{0}(f), H_{-4}(f)$ and $H_{4}(f)$, showing increased passband gain and reduced folding to the pass-band when including an inductor.

Using the functions $\mathrm{G}(\mathrm{f})$ and $\mathrm{I}(\mathrm{f})$, the Fourier transform can now be applied to equation (18) following a similar approach as [18], app. A and B. However, not only the Fourier transform of the capacitor voltage (eqn.77 in [18]), but also that of the derivative of the capacitor voltage is needed, for which we find:

$$
\begin{aligned}
& \sum_{n=-\infty}^{\infty} \mathcal{F}\left(\frac{d v_{c}(t)}{d t} \delta\left(t-n T_{s}-\sigma_{k}\right)\right) \\
& =\sum_{n=-\infty}^{\infty} I_{k}\left(f-n f_{s}\right) f_{s} e^{-\frac{j 2 \pi n}{N}} V_{i}\left(f-n f_{s}\right)
\end{aligned}
$$

We can then find the solution for one kernel. As all kernels are equal, their response is equal apart from a phase shift and hence calculating only $\mathrm{H}_{n, 1}$ is sufficient. In equation (10) we see that the polyphase transfer function will only produce results non-equal to zero when $\mathrm{n} / \mathrm{N}$ is an integer. Considering that $\sigma_{0}=$ 0 and $\sigma_{1}=\tau_{1}=\frac{T_{S}}{N}=\frac{1}{N f_{S}}$, after some math we get:

$$
\begin{aligned}
& H_{n, 1}(f) \\
& \quad=\frac{1}{-(2 \pi f)^{2}-j 2 \pi f B_{1}-A_{1}}\left(C_{1} \frac{1-e^{-j 2 \pi n f_{s} \tau_{1}}}{j 2 \pi n}\right. \\
& \quad+\left(-B_{1}+j 2 \pi f\right)\left(f_{s} G_{0}\left(f-n f_{s}\right)-f_{s} G_{1}\left(f-n f_{s}\right)\right) \\
& \left.\quad+f_{s} I_{0}\left(f-n f_{s}\right)-f_{s} I_{1}\left(f-n f_{s}\right)\right), \quad n / N=\text { integer }
\end{aligned}
$$

By using equation (10), filling in the correct coefficients as defined in (17) and taking into account that $I_{1}$ is equal to $I_{0}$, the polyphase transfer can be determined:

$$
\begin{aligned}
& H_{n, \text { polyphase }}(f) \\
& =H(f) \cdot N\left(\frac{1-e^{-j 2 \pi n f_{s} \tau_{1}}}{j 2 \pi n}+(R C+j 2 \pi f L C)\right. \\
& \left.\quad \cdot f_{s}\left(G_{0}\left(f-n f_{s}\right)-G_{1}\left(f-n f_{s}\right)\right)\right), \quad n / N=\text { integer } \\
& H_{n, \text { polyphase }}(f)=0, \quad n / N \neq \text { integer }
\end{aligned}
$$

\section{Model VerificAtion AND APpliCATION}

To verify the analysis, the transfer function was calculated and simulated for a 4-path filter with $\mathrm{f}_{\mathrm{s}}$ of $1 \mathrm{GHz}, \mathrm{R}$ of $50 \Omega, \mathrm{C}$ of $50 \mathrm{pF}$ and an inductance of 0 and $10 \mathrm{nH}$. Figure 5 shows the simulated and calculated curves are identical, confirming

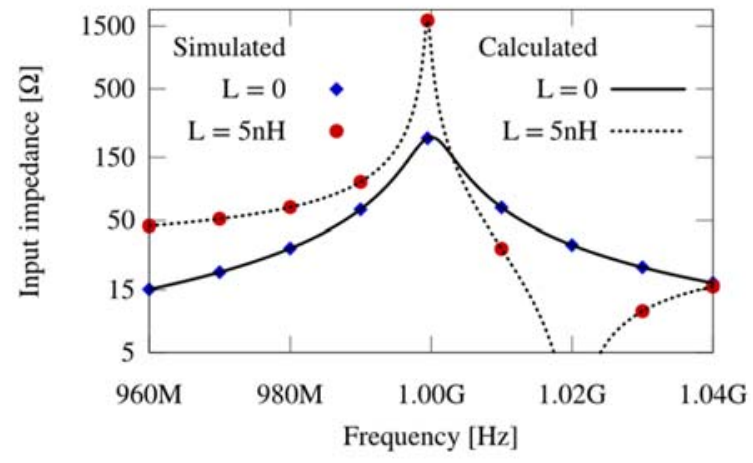

Figure 4 Input impedance evaluated at RF-frequencies close to $\mathrm{f}_{\mathrm{s}}=1 \mathrm{GHz}$

TABLE I

DEFAULT VALUES USED FOR SIMULATIONS

\begin{tabular}{ccl}
\hline \hline \multirow{2}{*}{ Symbol } & Quantity & Value \\
\hline $\mathrm{f}_{\mathrm{s}}$ & Switching frequency & $1 \mathrm{GHz}$ \\
$\mathrm{R}$ & Filter Resistance & $50 \Omega$ \\
$\mathrm{C}$ & Filter Capacitance & $50 \mathrm{pF}$ \\
$\mathrm{L}$ & Series inductance & $10 \mathrm{nH}$ or variable \\
& & as indicated
\end{tabular}

correct analysis. We will now compare some results for the resistive and inductive source impedance and show that adding an inductor can improve filter properties significantly. First the filter shape will be discussed in section A, then the input impedance (B), noise (C), harmonic response (D) and finally the unwanted signal folding in in sub-section $\mathrm{E}$.

\section{A. Filter Shape}

Substituting $n=0$ in equation (56) and taking the limit of the first term between brackets for $\mathrm{n}$ approaching zero, yields:

$$
\begin{aligned}
H_{0, \text { polyphase }}(f)=N \cdot H(f) & \quad\left(\frac{1}{N}+(R C+j 2 \pi f L C)\left(f_{s} G_{0}(f)-f_{s} G_{1}(f)\right)\right) \\
= & H(f)\left(1+\frac{1}{\Gamma(f)}\left(G_{0}(f)-G_{1}(f)\right)\right)
\end{aligned}
$$

with:

$$
\begin{gathered}
H(f)=\frac{1}{1+j 2 \pi f R C-(2 \pi f)^{2} L C} \\
\Gamma(f)=\frac{T_{s} / N}{R C+j 2 \pi f L C}=\frac{j 2 \pi f T_{s}}{N} \cdot \frac{H(f)}{1-H(f)}
\end{gathered}
$$

This equation is of the same form as equation (12), but $\mathrm{H}(\mathrm{f})$ is a second order low-pass function instead of a first order function and $\Gamma$ is now also a function of frequency $f$ due to the extra LC-term in the denominator. Actually, equation (58) reduces to a regular second order low-pass function when $\Gamma$ approaches infinity. However, this occurs when $T_{\mathrm{s}}$ goes to infinity, i.e. when the switching is eliminated and only one switch is permanently on. Hence, the first term between brackets represents low-pass behavior. The second term in equation (58) with $\mathrm{G}_{0}$ and $\mathrm{G}_{1}$ contains exponentials rendering repetitive peaks in the transfer characteristic at multiples of the switching frequency. To understand this intuitively, please refer to the time domain domain analysis in [5]. When $\Gamma$ decreases, 


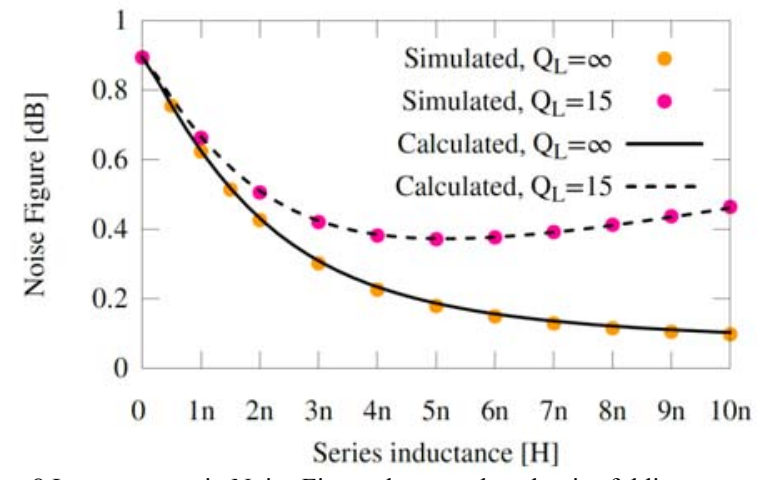

Figure 8 Improvement in Noise Figure due to reduced noise folding.

these peaks become narrower, i.e., quality factor increases. Compared to a resistive source impedance, there is an additional LC-term in the denominator of $\Gamma$, so that the series inductance renders an extra degree of freedom to adjust the filter. If the bandwidth of this filter is compared to that of a filter with resistive source impedance, it appears that the same bandwidth can be realized with a lower capacitance.

\section{B. Input Impedance}

We define an input impedance close to the fundamental as [5]:

$$
Z_{x, 0}=\frac{v_{x, 0}(f)}{i_{x, 0}(f)}=\frac{H_{0}(f) R+j 2 \pi f L}{1-H_{0}(f)}
$$

Figure 4 shows a plot of this impedance for two L-values. As $H_{0}(f)$ contains imaginary terms due the up-converted impedance of the baseband capacitors, there is a dip in impedance for $\mathrm{L}=5 \mathrm{nH}$, because the $\mathrm{R}$ - and $\mathrm{L}$-term in the numerator of eqn.(61) largely cancel each other at $1.02 \mathrm{GHz}$.

\section{Noise Figure}

The noise figure can be written as the total output noise divided by the output noise contribution from the source via the intended frequency translation:

$$
F=\frac{\sum_{n=-\infty}^{\infty} H_{n}(f)^{2}}{H_{0}(f)^{2}}
$$

Figure 8 shows that adding a series inductor improves the noise figure, as expected, due to the reduced noise folding. In practice finite inductor-Q will limit the improvement as is also shown.

\section{Harmonic Responses}

As can be seen in Figure 6, the N-path filter also has harmonic responses at $m f_{s}$. The gain at $2 f_{s}$. is almost equal to the gain at the switching frequency for higher values of the inductance. However, in a differential topology even harmonic responses can be cancelled.

Using equation (58) the filter response near harmonics of the switching frequency was analyzed versus inductance value. In Figure 7 , the absolute value of the peaks at a multiple $m$ of the switching frequency is represented as a function of the series inductance. The values are calculated for a 4-path filter with $\mathrm{f}_{\mathrm{s}}$ of $1 \mathrm{GHz}, \mathrm{C}$ of $50 \mathrm{pF}$ and $\mathrm{R}$ of $50 \Omega$. It is apparent from the graph that the gain in the pass band for $m=1$, i.e. the value of the peak at $f_{s}$, is a strongly increasing function of $L$ and can be much bigger than 1 . Note that this concerns voltage, not power gain. The second harmonic response even grows faster with

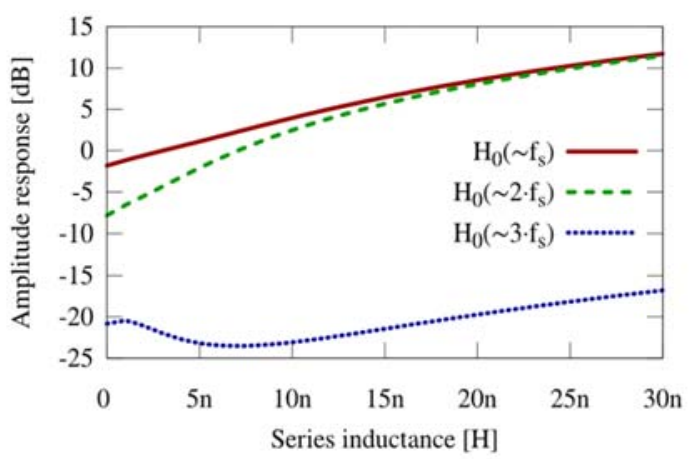

Figure 7 Calculated peak values of the transfer function near the fundamental and second and third harmonic.

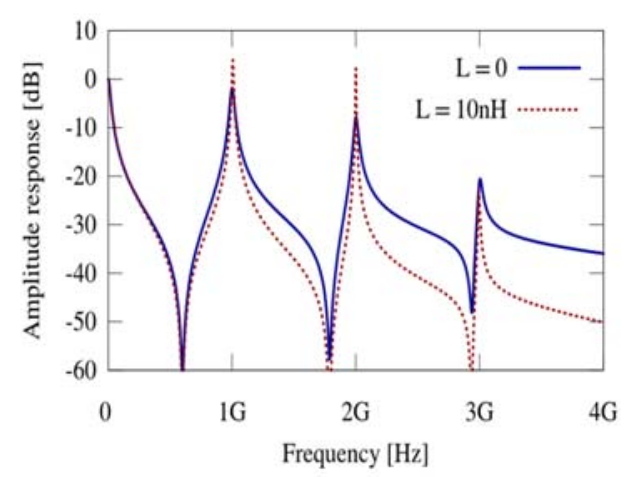

Figure 6 Amplitude of $\mathrm{H}_{0}(\mathrm{f})$ for a 4-path filter, using the values in Table I.

inductance, but can be cancelled in a fully differential topology. The third harmonic suppression compared to the fundamental benefits from increasing inductance especially for $1-10 \mathrm{nH}$.

\section{E. Effect of folding (aliasing)}

Next, the unwanted signal folding effects will be treated. The transfer of the N-path filter with $\mathrm{n}$ unequal to zero is given by:

$$
\begin{aligned}
& H_{n, \text { polyphase }}(f) \\
& \quad=H(f) \cdot \frac{1}{\Gamma(f)}\left(G_{0}\left(f-n f_{s}\right)-G_{1}\left(f-n f_{s}\right)\right), \\
& \quad n / N=\text { integer and } n \neq 0
\end{aligned}
$$

If $\mathrm{G}_{0}$ is evaluated for a frequency shifted input using equation (45), it appears that the only term dependent on $n f_{s}$ is $\mathrm{H}(\mathrm{f})$. For the remaining terms, the $\mathrm{n}$ dependency reduces to a phase shift of $2 \pi$ in the argument of the exponents. Using this result, the

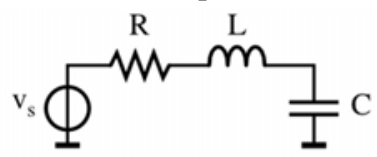

(a)

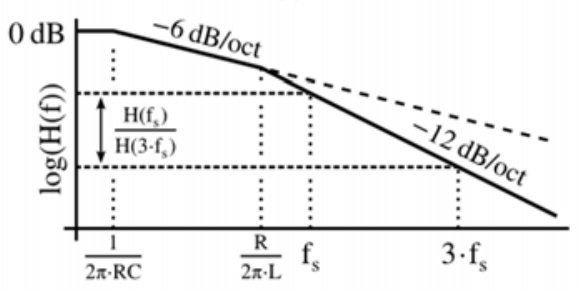

(b)

Figure 9 (a) Low-pass filter obtained by closing the switch of the kernel, (b) The resulting transfer function with the relevant rejection ratio. 
following relation can be written:

$$
G_{0}\left(f-n f_{s}\right)=G_{0}(f) \cdot \frac{H\left(f-n f_{s}\right)}{H(f)}
$$

If we also do this for $G_{1}$ and substitute in equation (63), we can calculate the ratio of a folded term to the wanted term, and using (58) we get:

$$
\frac{H_{n}(f)}{H_{0}(f)}=\frac{H\left(f-n f_{s}\right)}{H(f)} \cdot \frac{1}{1+\frac{\Gamma(f)}{G_{0}(f)-G_{1}(f)}},
$$$$
n / N=\text { integer and } n \neq 0
$$

If $\mathrm{G}_{0}-\mathrm{G}_{1}$ is sufficiently high and $\Gamma$ is small, the last term in equation (65) reduces to a value close to one. This assumption is true for N-path filters with a gain close to or above 1 at frequency $\mathrm{f}_{\mathrm{s}}$. The following approximation holds then:

$$
\frac{H_{n}\left(f_{s}\right)}{H_{0}\left(f_{s}\right)} \approx \frac{H\left(f_{s}-n f_{s}\right)}{H\left(f_{s}\right)}, \quad n / N=\text { integer }
$$

This is a very interesting outcome: it appears that the signal folding to $f_{s}$ is suppressed by low-pass filter $H(f)$. In other words, the suppression of the folded components will only be dependent on the low-pass network (with transfer function $H(f)$ ) that the input sees when the switch is closed (see Figure 9). This is also the case for an $\mathrm{N}$-path filter with resistive source impedance, because equations (12) and (15) are equivalent to equations (58) and (63). The only difference is that the function $\mathrm{H}(\mathrm{f})$ is now a second order low-pass function instead of a first order function.

This means that the suppression of components folding back to the desired band can be much higher. To quantify this effect, let's assume that both $f_{s}$ and $f_{s}-n f_{s}$ will fall outside the passband on the $-12 \mathrm{~dB} /$ octave slope of the low-pass function. Now, the ratio in equation (66) can be approximated by:

$$
\frac{H_{n}\left(f_{s}\right)}{H_{0}\left(f_{s}\right)} \approx\left(\frac{f_{s}}{f_{s}-n f_{s}}\right)^{2}, \quad \frac{n}{N}=\text { integer }
$$

The nearest component that folds back to the band of interest in a 4-path filter is the input shifted by $4 \mathrm{f}_{\mathrm{s}}(\mathrm{n}=4)$. Hence an RF signal at $3 f_{s}$ or $5 f_{s}$ is shifted to $f_{s}$ and falls on top of the filter band. As $3 f_{s}$ is closer to $f_{s}$ and hence more problematic, Figure 9b graphically shows the relevant "pre-filter" ${ }^{2}$ function $\mathrm{H}(\mathrm{f})$, which should now be evaluated at $\mathrm{f}_{\mathrm{s}}$ and $3 \mathrm{f}_{\mathrm{s}}$. Ratio $\mathrm{H}\left(3 \mathrm{f}_{\mathrm{s}}\right) / \mathrm{H}\left(\mathrm{f}_{\mathrm{s}}\right)$ gives the attenuation for the folding compared to the wanted signal at $\mathrm{f}_{\mathrm{RF}}=\mathrm{f}_{\mathrm{s}}$ (see Figure $9 \mathrm{~b}$, a rough estimate is $\left.(1 / 3)^{2}\right)$. Figure 10 plots the calculated folding versus L-value, both for the exact and approximate eqn. (67). Clearly, more folding reduction is usually wanted and one might wonder what happens for more paths. For an 8-path filter, the folding from $7 \mathrm{f}_{\mathrm{s}}$ is suppressed to roughly $(1 / 7)^{2}$ or $-34 \mathrm{~dB}$ (versus $-17 \mathrm{~dB}$ for $\mathrm{L}=0$ ). Still often, more attenuation is desired and extra low-pass pre-filtering should be added. From the equations derived in the previous section, it appears that the function $\mathrm{G}_{0}(\mathrm{f})$ is linearly related to the low-pass function $\mathrm{H}(\mathrm{f})$, both for a first order filter (resistive source impedance) and a second order filter (inductive source impedance). This H(f) dependency results from the particular solution of the differential equation. For a higher order pre-

\footnotetext{
${ }^{2}$ Strictly speaking this filter is not a separate pre-filter but an intrinsic part of the time variant circuit. Still, given our aim to reduce folding and
}

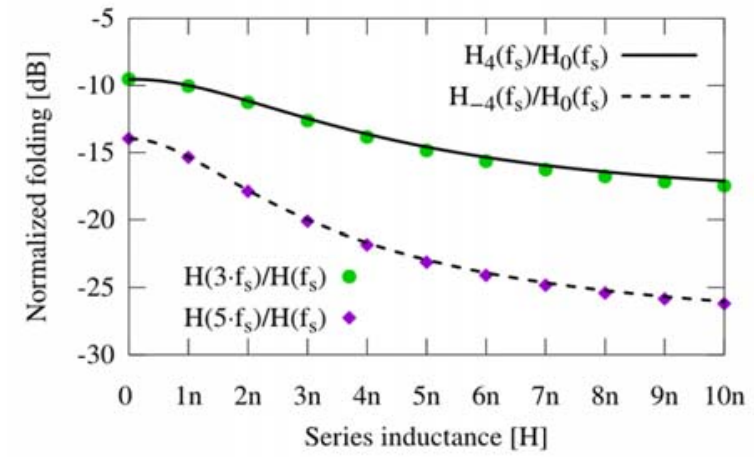

Figure 10 Calculated folding for $\mathrm{f}_{\mathrm{s}}=1 \mathrm{GHz}$ and variable $\mathrm{L}$, both eqn. (65) (lines) and approximation eqn. (66) (dots), showing reduction with increasing inductance.

filter, a higher order transfer function and more filtering will likely result, but demonstrating this is left as future work.

The folding from $3 f_{s}$ reduces from about -9 to $-17 d B$ when $L$ increases from 0 to $10 \mathrm{nH}$. In practice a fixed value of $\mathrm{L}$ will often be used, and then it may be interesting to see how the passband shape varies and how folding reduction varies as a function of the target center frequency $\mathrm{f}_{\mathrm{s}}$. The results are shown in Figure 11. It can be verified that the best passband gain and least (relative) folding from $3 \mathrm{f}_{\mathrm{s}}$ occurs at $2 \mathrm{GHz}$.

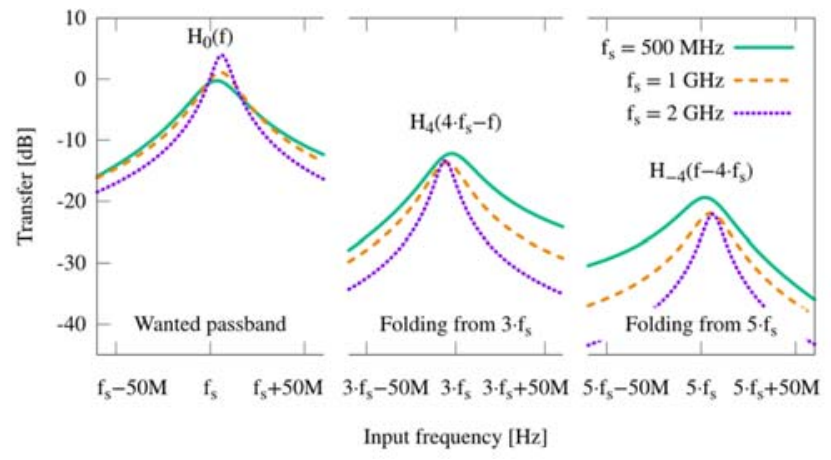

Figure 11. Calculated wanted passband and unwanted folding for a fixed series inductance of $5 \mathrm{nH}$ and $\mathrm{f}_{\mathrm{s}}$ equal to $0.5,1$ and $2 \mathrm{GHz}$.

It should be noted that the results above assume an ideal inductor. A practical inductor may show parasitic capacitance to ground, which may have detrimental effects. Further research is needed to quantify what is feasible in practice, where this paper derives the ideal limit. The model in Figure 9 with the "pre-filter" concept is intuitively attractive. However, it should be noted that it is an approximation which is only valid if $f_{s}$. $N \cdot R \cdot C>1$. Figure 12 illustrates model validity limits for $\mathrm{L}=5 \mathrm{nH}$, showing a good match above $200 \mathrm{MHz}$.

\section{CONCLUSIONS}

Analytical equations for the transfer function and signal folding of single-ended $\mathrm{N}$-path filters with resistive and inductive series impedance have been derived. The analysis assumes ideal switches that switch instantly from an onresistance of zero to an off-resistance of infinity. Furthermore,

given the filter transfer is multiplied with the N-path transfer function, we use this term. 


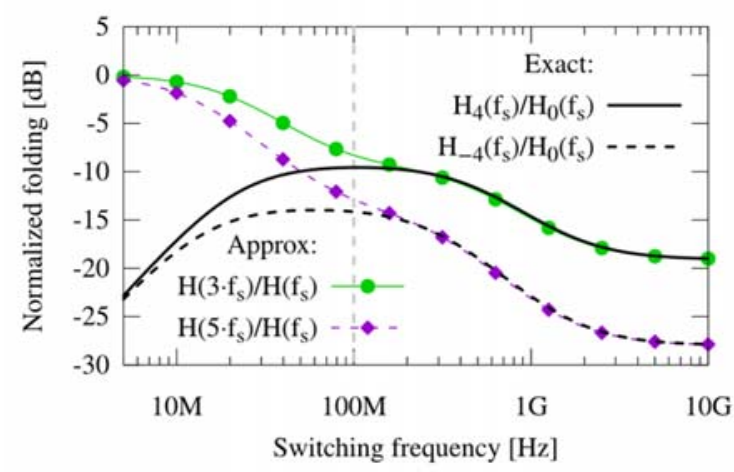

Figure 12 Calculated folding (exact and approximate (lines with markers)) with $L=5 \mathrm{nH}$, showing good approximation for $f_{S}>\frac{1}{N \cdot R \cdot C}(=100 \mathrm{MHz})$

the duty-cycle is assumed ideal, i.e. equal to $1 / \mathrm{N}$.

The resulting equations have been examined and show that the addition of a series inductance results in improved band pass filter behavior. Increasing this inductance increases the in-band voltage gain and reduces the bandwidth, while also reducing noise and odd order harmonic responses. Moreover, and even more important for realizing a practical N-path filter, the addition of an inductor increases the suppression of signal folding from $(\mathrm{N}-1) \mathrm{f}_{\mathrm{s}}$ to $\mathrm{f}_{\mathrm{s}}$. Interestingly, a good estimate for the filtering of folding products can be obtained by simply considering the low-pass filter that results when only one switch of the filter is continuously closed.

\section{APPENDIX A}

When looking at a single R-C kernel, during the on-time of the switch, the system is linear time invariant and the voltage is described by differential equation (1). Using step function $u(t)$, this equation can be adapted to one valid for all $t$ as follows:

$$
\begin{aligned}
& \frac{d v_{c}(t)}{d t} \sum_{n=-\infty}^{\infty}\left(u\left(t-n T_{s}-\sigma_{k-1}\right)-u\left(t-n T_{s}-\sigma_{k}\right)\right) \\
& =\left(A_{k, R} v_{c}(t)+C_{k, R} v_{i}(t)\right) \\
& . \sum_{n=-\infty}^{\infty}\left(u\left(t-n T_{s}-\sigma_{k-1}\right)-u\left(t-n T_{s}-\sigma_{k}\right)\right)
\end{aligned}
$$

This equation also defines $\mathrm{v}_{\mathrm{c}}$ during interval $\mathrm{k}$, i.e. $\mathrm{v}_{\mathrm{c}, \mathrm{k}}$. Since $\mathrm{V}_{\mathrm{c}, \mathrm{k}}$ is equal to $\mathrm{V}_{\mathrm{c}}$ within interval $\mathrm{k}$ and zero outside this interval, $\mathrm{v}_{\mathrm{c}, \mathrm{k}}$ can be written as:

$$
\begin{aligned}
& v_{c, k}(t) \\
& =v_{c}(t) \sum_{n=-\infty}^{\infty}\left(u\left(t-n T_{s}-\sigma_{k-1}\right)\right. \\
& \left.\quad-u\left(t-n T_{s}-\sigma_{k}\right)\right)
\end{aligned}
$$

For simplicity, during further calculations for one kernel the summation is left out in this expression and $n=0$ is substituted. In the end, the summation and time shift $n T_{s}$ will be reintroduced when combining to a polyphase system. Hence, the simplified $\mathrm{v}_{\mathrm{c}, \mathrm{k}}$ will be:

$$
v_{c, k}(t)=v_{c}(t)\left(u\left(t-\sigma_{k-1}\right)-u\left(t-\sigma_{k}\right)\right)
$$

The same can be done for $\mathrm{v}_{\mathrm{i}, \mathrm{k}}$ by simply replacing $\mathrm{v}_{\mathrm{c}}$ by $\mathrm{v}_{\mathrm{i}}$ in the above equation. Using the product rule, the derivative of $v_{c, k}$ can be calculated as well:

$$
\begin{aligned}
& \frac{d v_{c, k}(t)}{d t} \\
& \quad=\frac{d v_{c}(t)}{d t}\left(u\left(t-\sigma_{k-1}\right)-u\left(t-\sigma_{k}\right)\right) \\
& +v_{c}(t)\left(\delta\left(t-\sigma_{k-1}\right)-\delta\left(t-\sigma_{k}\right)\right)
\end{aligned}
$$

Applying the same simplifications to equation (68) yields:

$$
\begin{aligned}
& \frac{d v_{c}(t)}{d t}\left(u\left(t-\sigma_{k-1}\right)-u\left(t-\sigma_{k}\right)\right) \\
& \quad=\left(A_{k, R} v_{c}(t)+C_{k, R} v_{i}(t)\right)\left(u\left(t-\sigma_{k-1}\right)\right. \\
& \left.\quad-u\left(t-\sigma_{k}\right)\right)
\end{aligned}
$$

Using equations (70) and (71), equation (72) can easily be expressed in terms of $\mathrm{v}_{\mathrm{c}, \mathrm{k}}$ and $\mathrm{v}_{\mathrm{i}, \mathrm{k}}$ :

$$
\begin{aligned}
& \frac{d v_{c, k}(t)}{d t}-v_{c}(t)\left(\delta\left(t-\sigma_{k-1}\right)-\delta\left(t-\sigma_{k}\right)\right) \\
& \quad=A_{k, R} v_{c, k}(t)+C_{k, R} v_{i, k}(t)
\end{aligned}
$$

When the Dirac delta functions between brackets are replaced by the infinite sum of Dirac functions and time shift $n T_{s}$ is reintroduced, the result is equation (5).

For the R-L-C case, the equation that is valid for all $\mathrm{t}$, equivalent to equation (72) is as follows:

$$
\begin{aligned}
& \frac{d^{2} v_{c}(t)}{d t^{2}}\left(u\left(t-\sigma_{k-1}\right)-u\left(t-\sigma_{k}\right)\right) \\
& =\left(A_{k} v_{c}(t)+B_{k} \frac{d v_{c}(t)}{d t}+C_{k} v_{i}(t)\right) \\
& \cdot\left(u\left(t-\sigma_{k-1}\right)-u\left(t-\sigma_{k}\right)\right)
\end{aligned}
$$

As for the first order differential equation, the above equation should be expressed in terms of $v_{c, k}$. For this case, a second derivative of $\mathrm{v}_{\mathrm{c}, \mathrm{k}}$ is also needed:

$$
\begin{aligned}
& \frac{d^{2} v_{c, k}(t)}{d t^{2}} \\
& =\frac{d^{2} v_{c}(t)}{d t^{2}}\left(u\left(t-\sigma_{k-1}\right)-u\left(t-\sigma_{k}\right)\right) \\
& +\frac{d v_{c}(t)}{d t}\left(\delta\left(t-\sigma_{k-1}\right)-\delta\left(t-\sigma_{k}\right)\right) \\
& +\frac{d\left(\left(v_{c}(t)\left(\delta\left(t-\sigma_{k-1}\right)-\delta\left(t-\sigma_{k}\right)\right)\right)\right.}{d t}
\end{aligned}
$$

Then, using equations (70), (71) and (75), equation (74) can be rewritten as:

$$
\begin{aligned}
& \frac{d^{2} v_{c, k}(t)}{d t^{2}}-\frac{d v_{c}(t)}{d t}\left(\delta\left(t-\sigma_{k-1}\right)-\delta\left(t-\sigma_{k}\right)\right) \\
& \quad-\frac{d\left(\left(v_{c}(t)\left(\delta\left(t-\sigma_{k-1}\right)-\delta\left(t-\sigma_{k}\right)\right)\right)\right.}{d t} \\
& =A_{k} v_{c, k}(t) \\
& \quad+B_{k}\left(\frac{d v_{c, k}(t)}{d t}-v_{c}(t)\left(\delta\left(t-\sigma_{k-1}\right)-\delta\left(t-\sigma_{k}\right)\right)\right) \\
& +C_{k} v_{i, k}(t)
\end{aligned}
$$

For the N-path filter (76) reduces to equation (18) after reintroducing the infinite summation. 


\section{REFERENCES}

[1] H. Darabi, A. Mirzaei, and M. Mikhemar, "Highly Integrated and Tunable RF Front Ends for Reconfigurable Multiband Transceivers: A Tutorial," Circuits and Systems I: Regular Papers, IEEE Transactions on, vol. 58, pp. 2038-2050, 2011.

[2] A. Ghaffari, E. A. M. Klumperink, and B. Nauta, "A differential 4-path highly linear widely tunable on-chip band-pass filter," in Radio Frequency Integrated Circuits Symposium (RFIC), 2010 IEEE, 2010, pp. 299-302.

[3] A. Mirzaie, A. Yazdi, Z. Zhou, E. Chang, P. Suri, and H. Darabi, "A 65nm CMOS quad-band SAW-less receiver for GSM/GPRS/EDGE," in VLSI Circuits (VLSIC), 2010 IEEE Symposium on, 2010, pp. 179-180.

[4] C. Andrews and A. C. Molnar, "Implications of Passive Mixer Transparency for Impedance Matching and Noise Figure in Passive Mixer-First Receivers," Circuits and Systems I: Regular Papers, IEEE Transactions on, vol. 57, pp. 3092-3103, 2010.

[5] A. Ghaffari, E. A. M. Klumperink, M. C. M. Soer, and B. Nauta, "Tunable High-Q N-Path Band-Pass Filters: Modeling and Verification," SolidState Circuits, IEEE Journal of, vol. 46, pp. 998-1010, 2011.

[6] A. Mirzaei, H. Darabi, and D. Murphy, "A Low-Power Process-Scalable Super-Heterodyne Receiver With Integrated High-Q Filters," Solid-State Circuits, IEEE Journal of, vol. 46, pp. 2920-2932, 2011.

[7] A. Ghaffari, E. Klumperink, and B. Nauta, "8-Path tunable RF notch filters for blocker suppression," in Solid-State Circuits Conference Digest of Technical Papers (ISSCC), 2012 IEEE International, 2012, pp. 76-78.

[8] M. Darvishi, R. van der Zee, E. A. M. Klumperink, and B. Nauta, "Widely Tunable 4th Order Switched Gm-C Band-Pass Filter Based on N-Path Filters," Solid-State Circuits, IEEE Journal of, vol. 47, pp. 3105-3119, 2012.

[9] M. Darvishi, R. van der Zee, E. Klumperink, and B. Nauta, "A 0.3-to$1.2 \mathrm{GHz}$ tunable 4 th-order switched gm-C bandpass filter with $55 \mathrm{~dB}$ ultimate rejection and out-of-band IIP3 of $+29 \mathrm{dBm}, "$ in Solid-State Circuits Conference Digest of Technical Papers (ISSCC), 2012 IEEE International, 2012, pp. 358-360.

[10] A. Mirzaei, H. Darabi, and D. Murphy, "Architectural Evolution of Integrated M-Phase High-Q Bandpass Filters," Circuits and Systems I: Regular Papers, IEEE Transactions on, vol. 59, pp. 52-65, 2012.

[11] M. Darvishi, R. van der Zee, and B. Nauta, "Design of Active N-Path Filters," Solid-State Circuits, IEEE Journal of, vol. 48, pp. 2962-2976, 2013.

[12] Z. Lin, P. I. Mak, and R. P. Martins, "Analysis and Modeling of a GainBoosted N-Path Switched-Capacitor Bandpass Filter," Circuits and Systems I: Regular Papers, IEEE Transactions on, vol. PP, pp. 1-9, 2014.

[13] A. Ghaffari, E. A. M. Klumperink, F. E. van Vliet, and B. Nauta, "Simultaneous spatial and frequency-domain filtering at the antenna inputs achieving up to $+10 \mathrm{dBm}$ out-of-band/beam P1dB," in Solid-State Circuits Conference Digest of Technical Papers (ISSCC), 2013 IEEE International, 2013, pp. 84-85.

[14] A. Ghaffari, E. A. M. Klumperink, F. E. van Vliet, and B. Nauta, "A 4Element Phased-Array System with Simultaneous Spatial- and Frequency-Domain Filtering at the Antenna Inputs," Solid-State Circuits, IEEE Journal of (JSSC), vol. Accepted for publication, 2014.

[15] L. E. Franks and I. W. Sandberg, "An Alternative Approach to the Realization of Network Transfer Functions: The N-Path Filters," Bell Sys. Tech. J., vol. 39, pp. 1321-1350, 1960.

[16] L. Franks and F. Witt, "Solid-state sampled-data bandpass filters," in Solid-State Circuits Conference. Digest of Technical Papers. 1960 IEEE International, 1960, pp. 70-71.

[17] A. Ghaffari, E. A. M. Klumperink, and B. Nauta, "Tunable N-Path Notch Filters for Blocker Suppression: Modeling and Verification," Solid-State Circuits, IEEE Journal of, vol. 48, pp. 1370-1382, 2013.

[18] M. C. M. Soer, E. A. M. Klumperink, P. T. de Boer, F. E. van Vliet, and B. Nauta, "Unified Frequency-Domain Analysis of Switched-Series-RC Passive Mixers and Samplers," Circuits and Systems I: Regular Papers, IEEE Transactions on, vol. 57, pp. 2618-2631, 2010.

[19] A. El Oualkadi, M. El Kaamouchi, J. M. Paillot, D. VanhoenackerJanvier, and D. Flandre, "Fully Integrated High-Q Switched Capacitor Bandpass Filter with Center Frequency and Bandwidth Tuning," in Radio Frequency Integrated Circuits (RFIC) Symposium, 2007 IEEE, 2007, pp. 681-684.

[20] C. Andrews, L. Changhyuk, and A. Molnar, "Effects of LO harmonics and overlap shunting on N-phase passive mixer based receivers," in ESSCIRC (ESSCIRC), 2012 Proceedings of the, 2012, pp. 117-120.

[21] A. Mirzaei and H. Darabi, "Analysis of Imperfections on Performance of 4-Phase Passive-Mixer-Based High-Q Bandpass Filters in SAW-Less
Receivers," Circuits and Systems I: Regular Papers, IEEE Transactions on, vol. 58, pp. 879-892, 2011.

[22] A. Molnar and C. Andrews, "Impedance, filtering and noise in n-phase passive CMOS mixers," in Custom Integrated Circuits Conference (CICC), 2012 IEEE, 2012, pp. 1-8.

[23] T. Strom and S. Signell, "Analysis of periodically switched linear circuits," Circuits and Systems, IEEE Transactions on, vol. 24, pp. 531$541,1977$.

[24] M. Tenenbaum and H. Pollard, Ordinary Differential Equations: Dover Publications, 1985.

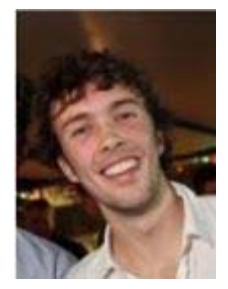

Lammert Duipmans received his MSc from the University of Twente in 2013. His MSc thesis deals with the suppression of harmonic folding effects in N-path filters by pre-filtering, and the analysis in this paper is largely based on his thesis.

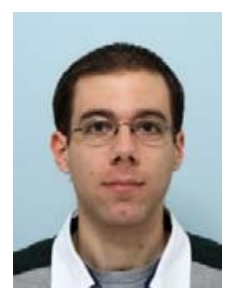

Remko Struiksma received his BEng degree from NHL University of Applied Sciences in 2006, and his MSc degree from the University of Twente. After his MSc, he joined the IC Design group of the University of Twente as research assistant. He research work focusses on radio receiver architectures and N-path filters and he currently works towards his $\mathrm{PhD}$.

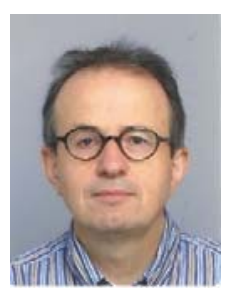

Eric A. M. Klumperink received his $\mathrm{PhD}$ in 1997 from the University of Twente in Enschede, The Netherlands and is currently an Associate Professor there with research focus on Radio Frequency ICs and Beamforming. Eric served as associate editor for IEEE TCAS-II, TCAS-I and JSSC, and is a TPC member of ISSCC and RFIC. He serves as IEEE Distinguished lecturer and is co-recipient of the 2002 and 2009 "ISSCC Van Vessem Outstanding Paper Award".

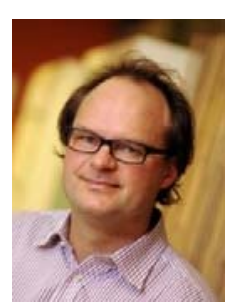

Bram Nauta received his $\mathrm{PhD}$ in 1991 from the University of Twente, Enschede, The Netherlands. After 7 years in Philips Research labs, he returned as full professor, heading the IC Design group. He served as TPC member of the major conferences in the field, was program chair of ISSCC and the editor-in-chief of IEEE J. Solid State Circuits. Bram is IEEE fellow, member of IEEE-SSCS AdCom and co-recipient of several best paper awards.

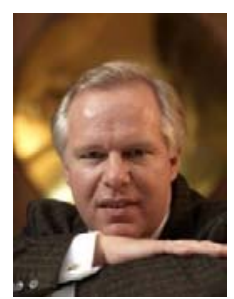

Frank van Vliet received his Ph.D. from Delft University of Technology, The Netherlands. He joined TNO (Netherlands Organization for Applied Scientific Research) in 1997, where he is currently a principal scientist responsible for MMIC, antenna and transmit \& receive module research. Since 2007 , he is also a professor in microwave integration in the ICD group of the University of Twente. Frank van Vliet (co-)authored well over 100 peer-reviewed publications. 
$* * * * * * * * *$ REPLY TO REVIEWERS ***********

Decision: Resubmit after Minor Revision for Review as a Regular Paper

Reviewers' and Associate Editor's Comments

$==$

The reviewers found that the manuscript is of great interest and significance for the topic of N-path filters. When the authors submit their revised manuscript, please address all reviewer's comments adequately. Thank you. Pui-In Mak.

A: We would like to thank the editors and reviewers for their useful review comments and quick response! Questions/Comments are indicated below with Q:(non-italic), and answers as: A: (italics).

Review Number 1.

$* * * * * * * * * * * * * * * * *$

Comments to the Author

Q: These are all minor comments, most are minor typos/imperfect English, as well as a few points that would benefit from clarification and/or some added intuitive explanation.

In abstract: grammer: This paper proofs $>>$ proves

A: Corrected

Q: only with the advent of nanometer CMOS technologies such filters can now operate at low $\mathrm{GHz}$ RF frequencies. $>>$ only with the advent of nanometer CMOS technologies can such filters now operate at low $\mathrm{GHz}$ RF frequencies.

\section{A: Corrected}

Q: However, filter design is less straightforward than as one may might expect.

\section{A: Corrected}

A: Unclear wording: "i.e. $\tau 1=\mathrm{Ts} / \mathrm{N}$ will its "track time", and $\tau 2=\mathrm{Ts}-\mathrm{Ts} / \mathrm{N}$ the "hold time","

A: To avoid confusion about track and hold terminology which is not used in the rest of the paper anyhow, we changed the text to: "..We choose the kernel for which the switch is closed during $k=1$ for analysis, so $\tau 1$ is the on-time of the switch and $\tau 2$ the offtime, as shown in Figure 3b."

Q: Hence, some of the harmonic folding terms remain un-cancelled,

\section{A: Corrected (still some >> some)}

Q: "For $\mathrm{L}=5 \mathrm{nH}$, the $\mathrm{R}$ - and L-term in the numerator largely cancel each other close to $1.02 \mathrm{GHz}$, explaining the impedance dip." This could be better explained, or supported by an additional intuitive explaination: you just have the basic impedance presented by the mixer (which includes imaginary terms from the up-converted impedance of the baseband capacitors) plus that of the inductor: if you show the real and imaginary parts separately, it becomes clear.

A: We tried to clarify this: "As $\mathrm{H}_{0}$ (f) contains imaginary terms due to the up-converted impedance of the baseband capacitors, there is a dip in impedance for $\mathrm{L}=5 \mathrm{nH}$, because the R- and L-term in the numerator largely cancel each other close to $1.02 \mathrm{GHz}$." We tried showing the real and imaginary parts in one graph, but don't feel this clarifies a lot, while it takes space and is not a key focus issue.

Q: "As can be seen in Figure 6, the N-path filter also has harmonic responses at ..fs. The gain at $2 \mathrm{fs}$. is almost equal to the gain at the switching frequency for higher values of the inductance. However, in a differential topology even harmonic responses can be cancelled." A bit of intuitive explanation of this result and that in Fig. 8 would be helpful...

A: To understand this intuitively, time-domain waveform are instructive as used in [5]. As these waveforms are less useful for this paper which is already overlength, we instead added a sentence: "To understand this intuitively, please refer to the time domain domain analysis in [5]."

we can calculate of the ratio of a folded term

A: Corrected (remove of)

Figure 12: It's a little confusing which curves are approximation and which are complete solutions: please be completely explicit! A: Corrected: we added "exact" and "approximate" as labels

\section{Review Number 2.}

$* * * * * * * * * * * * * * * * *$

Comments to the Author

Q: This paper is an extension of the previous works of authors in [17]. The analysis yields accurate result. However, that an inductor can be used to implement a switched N-path filter with a higher Q, thus improving the filtering has already been shown in the following paper,

El Oualkadi, A.; Paillot, J.-M.; Guegnaud, H.; Allam, Rachid, "A novel Q-enhanced LC switched-capacitor bandpass filter for digital wireless RF applications," Radio Frequency Integrated Circuits (RFIC) 
Symposium, 2004. Digest of Papers. 2004 IEEE, vol., no., pp.659,662, 6-8 June 2004.

This reference needs to be added.

A: We should indeed have referenced this paper as well as another [19,20].

The analysis is rigorous and the predicted values match well with the Spectre simulations.

The issues with the paper are the following:

Q1. In page 2, Fig. 2 legend, I think it should be

Simulated with 'L', not 'Ls'.

\section{A: Corrected}

Q2. Page 2, the paragraph before Fig. 3, the sentence "... by adding a phase shift term later in Subsection A" does not make much sense. Recommend rephrasing.

A: Changed to:

"First, the transfer of only one kernel will be analyzed, as other kernels have the same transfer function, except for a phase shift in the clock. We can find the combined effect of all kernels in a polyphase system, using the analysis for one kernel and adding that phase shift term later, in subsection A."

Q3. In the same paragraph " ... will its track time ..." does not convey any meaning.

A: To avoid confusion about track and hold terminology which is not used in the rest of the paper anyhow, we changed the text to: "..We choose the kernel for which the switch is closed during $k=1$ for analysis, so $\tau 1$ is the on-time of the switch and $\tau 2$ the off-time, as shown in Figure 3b."

Q4. Vc,k(f) and Hn,k,R(f) are not defined in Eq. (6). A: The text before and after Eq.(6) has been changed to define these variables more explicitly.

Q5. Eq. 7 is not new, it is re-derived from [17]. It should be added as a reference here.

A: Done

Q6. Page 3, the paragraph after Eq. (9), is it G0(f) or $\mathrm{G} 0 \mathrm{R}(\mathrm{f})$ ?

A: Both is correct, but we added the index $R$ also in the text to avoid unnecessary confusion.

Q7. While describing the analysis framework in Section II, the authors have used $\mathrm{Hn}$ and $\mathrm{Hn}, \mathrm{R}$ interchangeably in some places, e.g. in the paragraph after Eq. (11). More consistency is needed in usage of the notations.

$A$ : We consistently use index $R$ now in section $I I$, which discusses the purely resistive $(L=0)$ case.
Q8. Fig. 11 is tough to read.

A: Admittedly, intuitive interpretation may not be easy, but we don't see how to improve this.

Q9. Page 4, Section III, paragraph 2, the line before Eq. 17 , there are two "now"-s.

A: Removed

Q10. Page 6, paragraph before Eq. (54), ".. but also of the derivative of the ... " two "of"-s.

A: Actually there should be two times and "of" because a Fourier transform is meant. We added the word "that" to more clearly refer back to the Fourier transform.

Q11. Page 7, the paragraph following Eq. (61), "... in the numerator ..." the Eq. number is missing.

$A$ : Changed to "because the R- and L-term in the numerator of eqn.(61) largely cancel each other at 1.02 $\mathrm{GHz}$.

Q12. Fig. 7, I am not sure why the $\mathrm{QL}=$ infinity case is shown. For on-chip inductors, QL is never infinity. All the other plots in this paper compare the performance of switched $\mathrm{RC}(\mathrm{L}=0$, so $\mathrm{QL}=0)$ and switched $\mathrm{RLC}$ filter (with a finite $\mathrm{QL}$ ).

A: As used in our noise analysis, the resistance $R$ is the source resistance, and can for instance represent the antenna impedance to which the noise figure is referred. Although other resistance, e.g. switch resistance and inductor series resistance can be "shifted out" and combined with $R$ to calculate the current in a seriesswitch-R-C circuit, for noise figure analysis it makes a difference whether the resistance is part of the signal source, or a parasitic resistance of other $\mathrm{N}$-path filter components. Hence we show one curve to fit to the analysis ( $Q L=$ infinity) and one, that gives an idea of a practical limitation with $Q L=15$. Note that the inductor may also be put off-chip and realized in the antenna-tochip interface. In the original paper nothing was said about the $Q L=15$ case, which admittedly is confusing. We tried to improve it by stating:

"Figure 6 shows that adding a series inductor improves the noise figure, as expected, due to the reduced noise folding. In practice finite inductor-Q will limit the improvement as is also shown."

Q13. Page 7, Section IVD, "... power gain is obviously not possible from a passive circuit ..." is not necessarily true. Passive LTI circuits don't give power gain.

However, non-linear or time-varying linear passive circuits can cause power gain through a parametric power amplification mechanism.

For example see the following references: 
Parametric Signal Amplification in Continuous Time Domain, http://www.springer.com/cda/content/document/cda downloaddocument/9781461416708-c1.pdf?SGWID =00-45-1268752-p174196973

A: Thanks for spotting this too strong claim. We removed it and state now: "Note that this concerns voltage, not power gain."

Q14. In page 8, paragraph 1, the quantity should be "G0G1", not "G0 minus G1".

A: Corrected

Q15. In Page 8, paragraph 1, the statement, "decent filter response" is unclear to me. Does it mean (sub) unity gain ?

A: We removed the term "decent", as it adds no useful information and only factually specify gain.

\section{Review Number 3.}

$* * * * * * * * * * * * * * * * *$

Comments to the Author

This paper extends the known R-C N-path analysis to the R-L-C case. The derivation is clear and complete, although somewhat similar to [17]. Anyway, the analysis is good and the results by adding an inductor to the traditional N-path are interesting. However, it needs minor revision to improve the quality/clarity of the manuscript.

Here are my suggestions:

Q1) The following recently published N-path BPF have many good properties. Please add to the introduction to make it more complete.

Zhicheng Lin, P.-I. Mak, and R. P. Martins "Analysis and Modeling of a Gain-Boosted N-Path Switched-

Capacitor Bandpass Filter," IEEE Trans. Circuits Syst. I, Reg. Papers, 2014. doi: 10.1109/TCSI.2014.2312476.

A: We were not aware of this paper, but think it is indeed a very useful recent reference. We added it and refer twice to it.

Q2) In the introduction, there should be a "space" at the beginning of each section.

\section{A: Corrected}

Q3) Left hand column of page 2, please clarify what filter properties have been analyzed by using approximations in $[4,18,19]$. What is the difference between this analysis and that from $[4,18,19]$.

A: This has been clarified by the following text:

"The purpose of this paper is to derive analytical equations describing this interaction and its impact on signal transfer and folding. Although it has been observed that adding a series inductor can improve the filter transfer and noise [19, 20], only approximate equations for the desired transfer function, the input impedance and noise are available [4, 21, 22], assuming the RF-frequency is $f_{R F}=f_{S}+\Delta f$, with $\Delta f \ll f_{L O}$. Broadband signal folding cannot be analyzed with these equations."

Q4) A large inductor at the input of the mixer switches will definitely induce some parasitic capacitance, which can drastically shift the center frequency of the filter away from the LO clock, lowering in-band gain and increasing harmonic folding noise. Please discuss this issue in the paper.

A: What happens exactly when combined with an inductor is beyond the scope of this work, and we don't have equations that describe this behaviour (this requires solving a third order differential equation). Still we added the following text: "It should be noted that the results above assume an ideal inductor. A practical inductor may show parasitic capacitance to ground, which may have detrimental effects. Further research is needed to quantify what is feasible in practice, where this paper derives the ideal limit."

Q5) Left hand column of page 4, "assuming an input voltage equal to .., equation (16) is :", please put the reference such as book here.

A: We added a reference [24] and explain better now how the equation is solved (extra text including reference to specific chapters in [24])

Q6) Right hand column of page 5, "Using (45)" should be using (44), please correct that.

\section{A: Corrected}

Q7) In Appendix A, Ak, R and $\mathrm{Ck}, \mathrm{R}$ in equation (68) are not consistent with the rest of others equations $(\mathrm{Ak}, \& \mathrm{Ck})$, please try to correct that and make them consistent.

$A$ : Thanks, indices $R$ have been added up to and including eqn. (77) (resistive source part) 